The inequality

$$
\left|P_{k}\left(z_{1}, \cdots, z_{n}\right)\right| \leqq P_{k}\left(p_{1}, \cdots, p_{n}\right) \quad\left(\left|z_{1}\right| \leqq p_{1}, \cdots,\left|z_{n}\right| \leqq p_{n}\right),
$$

shows that (18) implies that $f \equiv 0$.

\title{
REFERENCES
}

1. E. Laguerre, Sur les fonctions du genre zéro et du genre un, Oeuvres de Laguerre, vol. 1, Paris, 1898, pp. 174-177.

2. G. Pólya, Über Annäherung durch Polynome mit lauter reellen Wurzeln, Rendiconti di Palermo vol. 36 (1913) pp. 1-17.

University of California, Los ANgeles, and

University of Pennsylvania

\section{ON THE EXISTENCE OF GREEN'S FUNCTION}

PETER D. LAX

In this note we shall present a very short proof of the existence of Green's function for Laplace's equation for any domain with sufficiently smooth boundary in any number of independent variables. The proof is based on the continuous dependence of solutions of Laplace's equation on their boundary values. It is a modification of a proof given by Paul Garabedian, see [1]; the difference between the two approaches is that whereas Garabedian operates with a representation of harmonic functions in terms of their boundary data which he obtains by a variational argument, in our argument only the linear and bounded dependence of the solution on the boundary values figures.

1. In this section we shall treat the somewhat simpler two-dimensional case.

We consider a bounded domain $D$ whose boundary $C$ consists of a finite number of smooth curves (i.e., curves with continuous tangents).

$B$ is the Banach space of all continuous functions defined on $C$, normed by the maximum norm.

$B^{\prime}$ is the submanifold of those elements of $B$ for which the boundary value problem can be solved. ${ }^{1}$

Received by the editors December 13, 1951.

${ }^{1}$ It is easy to show that $B^{\prime}$ is closed, but this is not necessary for the argument. 
Let $P$ be any arbitrary point of $D$. Associated with $P$ is a linear functional $L_{P}$ defined over $B^{\prime}$ :

$$
L_{P}\left[\phi^{\prime}\right]=h(P)
$$

where $h$ is the harmonic function whose value on $C$ is $\phi^{\prime} . L_{P}$ is clearly a linear functional, and by the maximum principle it is bounded, its bound being exactly one.

By the Hahn-Banach extension theorem, see [2], $L_{P}$ can be extended as a bounded linear functional to the whole space $B$; and we imagine it so extended. ${ }^{2}$

Consider then the following two-parameter family of elements in $B$ :

$$
\psi(\xi, \eta)=\psi(Q)=\log |z-Q|, \quad z \in C ;
$$

the point $Q=(\xi, \eta)$ may lie anywhere off the boundary $C$.

Clearly $\psi(\xi, \eta)$ has derivatives of second (in fact of all) order with respect to $\xi$ and $\eta$, and is harmonic with respect to $Q$ :

$$
\psi_{\xi \xi}+\psi_{\eta \eta}=0 \text {. }
$$

We construct the function

$$
k_{P}(Q)=L_{P}[\psi(Q)] .
$$

The operations of differentiating with respect to $\xi, \eta$ and applying the bounded linear functional $L_{P}$ commute. Therefore $k_{P}(Q)$ is a harmonic function, i.e., harmonic in each of the components of the complement of $C$.

Let $Q$ lie outside of $D$; the element $\psi(Q)$ lies in $B^{\prime}$, being the boundary value of the regular harmonic function $\log |z-Q|$. So

$$
k_{P}(Q)=L_{P}[\psi(Q)]=\log |P-Q| \quad \text { for } Q \text { not in } D .
$$

This explicit formula shows that $k_{P}(Q)$ is continuous up to the boundary in the exterior components. Next we shall show that the same is true as $Q$ approaches the boundary from the interior, and in fact we shall show that $k_{P}(Q)$ is continuous across $C$. This would mean that $k_{P}(Q)$ in $D$ is a harmonic function continuous up to the boundary and is equal to $\log |P-Q|$ there, i.e., $k_{P}(Q)$ is the regular part of Green's function.

As to the continuity of $k_{P}(Q)$ across $C$ : Let $Q$ be any point in $D$, $Q^{\prime}$ its mirror image with respect to the tangent at the nearest boundary point. If $Q$ is near enough to the boundary, $Q^{\prime}$ will lie outside of $D$; furthermore on account of the smoothness of $C$ the quotient

2 It should be pointed out that since $B$ is a separable Banach space, the extension can be accomplished without recourse to transfinite induction. 


$$
\frac{|z-Q|}{\left|z-Q^{\prime}\right|}
$$

tends to one uniformly for all $z$ on $C$ as the distance of $Q$ from the boundary shrinks to zero. Hence

$$
\max _{z \text { on } C}\left|\log \frac{|z-Q|}{\left|z-Q^{\prime}\right|}\right|=\left\|\psi(Q)-\psi\left(Q^{\prime}\right)\right\|
$$

tends to zero as $Q$ approaches $C$. Since $L_{P}$ is a bounded linear functional,

$$
k_{P}(Q)-k_{P}\left(Q^{\prime}\right)=L_{P}\left[\psi(Q)-\psi\left(Q^{\prime}\right)\right]
$$

also tends to zero as $Q$ approaches $C$.

This completes the proof for the plane; now we pass on to higher dimensions. The scheme of things remains the same; there is, however, some trouble in showing that $k_{P}(Q)$ is continuous across $C$ for it is no longer true that

$$
\psi(Q)-\psi\left(Q^{\prime}\right)=\frac{1}{|z-Q|^{n-2}}-\frac{1}{\left|z-Q^{\prime}\right|^{n-2}}
$$

tends to zero uniformly for all $z$ on $C$ as $Q$ approaches the boundary. This difficulty will be overcome by using a more refined property of $L_{P}$ than mere boundedness, namely, its monotonicity. According to the maximum theorem $L_{P}\left[\phi^{\prime}\right]$ is monotone over $B^{\prime}$, i.e., $L_{P}\left[\phi^{\prime}\right]$ $\geqq L_{P}\left[\phi^{\prime \prime}\right]$ if $\phi^{\prime} \geqq \phi^{\prime \prime} .^{3}$ What we shall show is that $L_{P}$ can be extended to $B$ without losing this property of monotonicity. The proof of this relies on the full statement of the Hahn-Banach theorem:

Let $L[\phi]$ be a bounded linear functional defined on the submanifold $B^{\prime}, N(\phi)$ a subadditive, positive homogeneous functional defined over the whole space $B$ which exceeds $L$ over $B^{\prime}$ :

$$
L[\phi] \leqq N(\phi)
$$

over $B^{\prime}$;

Then there exists an extension of $L$ to the full space which stays below $N$ :

$$
L[\phi] \leqq N[\phi]
$$

over $B$.

Our choice of $N[\phi]$ is as follows:

$$
N[\phi]=\operatorname{lub} L_{P}\left[\phi^{\prime \prime}\right], \quad \phi^{\prime \prime} \in B^{\prime}, \quad \phi^{\prime \prime} \leqq \phi .
$$

\footnotetext{
on $C$.

3 The symbol $\phi^{\prime} \geqq \phi^{\prime \prime}$ is defined to mean that $\phi^{\prime}-\phi^{\prime \prime}$ is a non-negative function
} 
$N[\phi]$, being the lub of linear functionals, is subadditive. It is clearly positive-homogeneous.

Since $L_{P}$ is monotone over $B^{\prime}, L_{P}=N$ over $B$. So, according to the Hahn-Banach theorem, $L_{P}[\phi]$ can be extended to the full space, linearly, so that it never exceeds $N[\phi]$.

To verify that $L_{P}$ thus extended is monotone it is sufficient to show that $L_{P}[\phi]$ is nonpositive if $\phi$ is nonpositive on $C$. Now $N[\phi]$ itself is monotone, as is easily seen from its definition (2); for nonpositive $\phi$, the handy sequence of inequalities

$$
L_{P}[\phi] \leqq N[\phi] \leqq N[0]=0
$$

holds and constitutes a proof of the result desired.

We define as before

$$
\psi(Q)=\frac{1}{|z-Q|^{n-2}}
$$

and construct $k_{P}(Q)=L_{P}[\psi(Q)] . k_{P}(Q)$ is harmonic in $D$; what remains to be shown is that it is equal to $|Q-P|^{-n+2}$ on the boundary of $D$.

We have to impose a further restriction on the boundary: that through each boundary point we can pass two spheres of radius $d$, one lying entirely within $D$, the other outside of $D$, with the same constant $d$ for all boundary points. ${ }^{4}$

Let $Q$ be some point of $D, R$ the nearest boundary point, and $K^{\prime}$ and $K^{\prime \prime}$ the interior and exterior tangent spheres through $R$. Let $Q^{\prime}$ and $Q^{\prime \prime}$ denote the inverted image of $Q$ with respect to $K^{\prime}$ and $K^{\prime \prime}$. If $Q$ is close enough to the boundary, $Q^{\prime}$ and $Q^{\prime \prime}$ are in the exterior.

Inverse points have the well known property that the quotient $|z-Q| /\left|z-Q^{\prime}\right|$ has some constant value $s^{\prime}$ for all points $z$ on the circumference of the circle $K^{\prime}$. The value of $s^{\prime}$ approaches unity as $Q$ tends to the boundary since the radius of $K^{\prime}$ does not change. Furthermore, if $z$ is any point outside of $K^{\prime},|z-Q| /\left|z-Q^{\prime}\right|$ is greater than $s^{\prime}$. This is in particular the case for $z$ lying on $C$ :

$$
s^{\prime} \leqq \frac{|z-Q|}{\left|z-Q^{\prime}\right|}
$$

for $z$ on $C$

which implies that

$$
\frac{s^{\prime n-2}}{|z-Q|^{n-2}} \leqq \frac{1}{\left|z-Q^{\prime}\right|^{n-2}}
$$

for $z$ on $C$.

- This kind of restriction on domains is familiar in potential theory. 
We rewrite the above as

$$
\psi(Q) \leqq \frac{1}{s^{\prime n-2}} \psi\left(Q^{\prime}\right)
$$

The monotone character of $L_{P}$ permits us to apply $L_{P}$ to both sides of (3) and retain the inequality:

$$
L_{P}[\psi(Q)] \leqq \frac{1}{s^{\prime n-2}} L_{P}\left[\psi\left(Q^{\prime}\right)\right] .
$$

By definition of the function $k_{P}$ this can be written as

$$
k_{P}(Q) \leqq \frac{1}{s^{\prime n-2}} k_{P}\left(Q^{\prime}\right)
$$

As in the two-dimensional case $k_{P}\left(Q^{\prime}\right)$ can be evaluated explicitly since $Q^{\prime}$ is not in $D$ :

$$
k_{P}\left(Q^{\prime}\right)=\frac{1}{\left|P-Q^{\prime}\right|^{n-2}} .
$$

So our inequality becomes

$$
k_{P}(Q) \leqq \frac{1}{s^{\prime n-2}} \frac{1}{\left|P-Q^{\prime}\right|^{n-2}} .
$$

The reverse inequality holds for $Q^{\prime \prime}$ :

$$
\frac{1}{s^{\prime \prime n-2}} \frac{1}{\left|P-Q^{\prime \prime}\right|^{n-2}} \leqq k_{P}(Q) \text {. }
$$

As $Q$ tends to some boundary point $R$, so does $Q^{\prime}$ and $Q^{\prime \prime}$, and $s^{\prime}$ as well as $s^{\prime \prime}$ tend to one. Thus the upper and lower bounds for $k_{P}(Q)$ both tend to $1 /|P-R|^{n-2}$, and $k_{P}(Q)$ itself cannot do otherwise.

I should like to mention that $C$. Miranda [3] has made use of the extension theorem for this problem in a different manner; his reasoning is slightly more delicate but then it gives more: the solvability of the boundary value problem for all continuous boundary data, not just the existence of Green's function. A similar reasoning plays a role in his treatment [4] of the first boundary value problem for the biharmonic equation; in this case of course the bounded dependence of the solution on the boundary data lies much deeper.

G. Fichera has made use of an equivalent of the Hahn-Banach theorem in his investigations on boundary value problems, see [5] and [6], and so has J. Deny in [7]. M. Brelot, in [8], utilized the possibility 
of monotonic extension of monotonic linear transformations to prove that the generalized solution, in Wiener's sense, of the first boundary value problem for Laplace's equation can be characterized as the least (resp. greatest) harmonic majorant (resp. minorant) of harmonic functions which are continuous up to the boundary and whose boundary values are less (resp. greater) than the prescribed data.

\section{BIBLIOGRAPHY}

1. P. R. Garabedian, The classes $L_{P}$ and conformal mapping, Trans. Amer. Math. Soc. vol. 69 (1950) pp. 392-415.

2. S. Banach, Théorie des opérations linéaires, Warsaw, 1932.

3. C. Miranda, Sul principio di Dirichlet per le funzioni armoniche, Atti della Accademia Nazionale dei Lincei. Rendiconti. Classe di Scienze Fisiche, Matematiche e Naturali (8) vol. 3 (1947) pp. 55-59.

4. - Formule di maggiorazione e teorema di esistenza per le funzioni biharmoniche di due variabli, Giornale di Matematiche di Battaglini (4) vol. 2 (1948) pp. 97-118.

5. G. Fichera, Teoremi di completezza sulla frontiera di una dominio per taluni sistemi di funzioni, Atti della Accademia Nazionale dei Lincei. Rendiconti. Classe di Scienze Fisiche, Matematiche e Naturali (8) vol. 3 (1947) pp. 502-507.

6. - - Teoremi di competezza sulla frontiera di un dominio per taluni sistemi di funzioni, Annali di Matematica Pura ed Applicata (4) vol. 27, pp. 1-28.

7. J. Deny, Sur l'approximation des fonctions harmoniques, Bull. Soc. Math. France vol. 73 (1945) pp. 71-73.

8. M. Brelot, Remarque sur le prolongement fonctionnel linéaire et le problème de Dirichlet.

New YoRK UNIversity 